\title{
Existence of the fundamental solution of a second order evolution equation
}

\author{
by JAN BOCHENEK (Kraków)
}

Wtodzimierz Mlak in memoriam

\begin{abstract}
We give sufficient conditions for the existence of the fundamental solution of a second order evolution equation. The proof is based on stable approximations of an operator $A(t)$ by a sequence $\left\{A_{n}(t)\right\}$ of bounded operators.
\end{abstract}

1. Introduction. Let $X$ be a real Banach space. Suppose that for each $t \in[0, T]$ a linear (in general unbounded) operator $A(t): X \rightarrow X$ is defined.

We make the assumption

$\left(\mathrm{Z}_{1}\right) \quad D(A):=D(A(t))$ is independent of $t, D(A)$ is dense in $X$ and for each $t \in[0, T]$ the operator $A(t)$ has a bounded inverse $A^{-1}(t)$.

Suppose that the operator $A(t)$ is strongly continuous on $D(A)$, i.e. for every $x \in D(A)$ the function $t \rightarrow A(t) x$ is continuous. It follows that the operator $A(t) A^{-1}(0)$ is bounded and continuous in $t$ on $[0, T]$, so in view of the Banach-Steinhaus theorem it is uniformly bounded in $t$, i.e.

$$
\left\|A(t) A^{-1}(0)\right\| \leq c \quad \text { for } t \in[0, T]
$$

(cf. $[7$, p. 9]).

If we assume that $A^{-1}(t)$ is uniformly bounded on $[0, T]$ then the mapping

$$
[0, T] \ni t \rightarrow A^{-1}(t)
$$

is strongly continuous. Analogously, if we assume that

$$
\left\|A(0) A^{-1}(s)\right\| \leq M \quad \text { for } 0 \leq s \leq T,
$$

then it will follow from the foregoing that the mapping

1991 Mathematics Subject Classification: 34G10, 47D06, 47D09.

Key words and phrases: evolution problem, stable family of operators, stable approximations of the evolution operator, fundamental solution, Cauchy problem, uniformly correct Cauchy problem. 


$$
[0, T] \times[0, T] \ni(t, s) \rightarrow A(t) A^{-1}(s)
$$

is strongly continuous (cf. [7, p. 177]).

We shall need the following.

Lemma 1 ([7, Lemma 1.5, p. 179]). Suppose that $A(t)$ is strongly continuously differentiable on its domain $D(A)$ and has a bounded inverse $A^{-1}(t)$. Then

$1^{\circ}$ The operator $A(t) A^{-1}(s)$ is continuous in the operator norm in the variables $s$ and $t$ taken together, where $0 \leq s, t \leq T$, and satisfies a Lipschitz condition in each of them. In particular, (3) holds.

$2^{\circ}$ The operator $A(t) A^{-1}(s)$ is strongly differentiable relative to $t$ and $s$, and the derivatives

$$
\left[A(t) A^{-1}(s)\right]_{t}^{\prime}=A^{\prime}(t) A^{-1}(s)
$$

and

$$
\left[A(t) A^{-1}(s)\right]_{s}^{\prime}=-A(t) A^{-1}(s) A^{\prime}(s) A^{-1}(s)
$$

are strongly continuous as functions of two variables $s$ and $t$.

The aim of this paper is to investigate the abstract second order linear initial value problem

$$
\frac{d^{2} u}{d t^{2}}=A(t) u+f(t), \quad t \in[0, T], \quad u(0)=u_{0}, \quad u^{\prime}(0)=u_{1},
$$

where $A(t)$, for $t \in[0, T]$, is the operator defined above, $u$ and $f$ are functions from $\mathbb{R}$ into $X$, and $u_{0}, u_{1} \in X$.

First we shall consider the first order problem.

2. First order initial value problem. In this section we consider the homogeneous differential equation

$$
\frac{d x}{d t}=A(t) x, \quad 0 \leq t \leq T,
$$

where $A(t)$ is the operator defined in the introduction and satisfying assumption $\left(\mathrm{Z}_{1}\right)$.

Definition 1. By a Cauchy problem for the equation (8) in the triangle $\Delta_{T}:=\{(s, t): 0 \leq s \leq t \leq T\}$ we mean the problem of finding for each fixed $s \in[0, T)$ a solution $x(t, s)$ of (8) on the segment $[s, T]$, satisfying a given initial condition

$$
x(s, s)=x_{0} \in D(A) .
$$

Definition 2 ([7, p. 193]). The Cauchy problem (8), (9) is said to be uniformly correct if: 
$1^{\circ}$ For each $s \in[0, T]$ and any $x_{0} \in D(A)$ there exists a unique solution $x(t, s)$ of $(8)$ on the segment $[s, T]$ satisfying condition (9).

$2^{\circ}$ The function $\Delta_{T} \ni(t, s) \rightarrow x(t, s)$ and its derivative $\Delta_{T} \ni(t, s) \rightarrow$ $x_{t}^{\prime}(t, s)$ are continuous.

$3^{\circ}$ The solution depends continuously on the initial data in the sense that if the $x_{0 n} \in D(A)$ converge to zero then the corresponding solutions $x_{n}(t, s)$ converge to zero uniformly relative to $(t, s) \in \Delta_{T}$.

It is known (see [7, p. 195]) that if the mapping $[0, T] \ni t \rightarrow A(t)$ is strongly continuous on $D(A)$ and the Cauchy problem (8), (9) is uniformly correct, then it is possible to introduce a linear operator $U(t, s): X \rightarrow X$ for $(t, s) \in \Delta_{T}$ which has the following properties:

$1^{\circ}$ The operator $U(t, s)$ is bounded in $X$ relative to $(t, s) \in \Delta_{T}$, i.e.,

$$
\|U(t, s)\| \leq M \quad(0 \leq s \leq t \leq T) .
$$

$2^{\circ}$ The mapping $\Delta_{T} \ni(t, s) \rightarrow U(t, s)$ is strongly continuous.

$3^{\circ}$ The following identities hold:

$$
U(t, s)=U(t, r) U(r, s), \quad U(t, t)=I \quad(0 \leq s \leq r \leq t \leq T) .
$$

$4^{\circ}$ On $D(A)$ the mapping $\Delta_{T} \ni(t, s) \rightarrow U(t, s)$ is strongly differentiable relative to $t$ and $s$, and

$$
\partial U(t, s) / \partial t=A(t) U(t, s), \quad \partial U(t, s) / \partial s=-U(t, s) A(s) .
$$

$5^{\circ}$ If, additionally, the operator $A(t)$ has a bounded inverse such that condition (3) is satisfied, then the operator

$$
V(t, s)=A^{-1}(t) U(t, s) A^{-1}(s)
$$

is bounded and strongly continuous in the triangle $\Delta_{T}$.

Definition 3. The operator $U(t, s)$, with $(t, s) \in \Delta_{T}$, having the properties $1^{\circ}-4^{\circ}$ is called the evolution operator (or fundamental solution) corresponding to equation (8).

Consider the family of equations

$$
\frac{d x}{d t}=A_{n}(t) x, \quad n=1,2, \ldots, t \in[0, T],
$$

with bounded strongly continuous operators $A_{n}(t)$.

Definition 4 ([7, p. 199]). If there exists a sequence of bounded strongly continuous operators $A_{n}(t)$ for which

$$
\lim _{n \rightarrow \infty}\left\{\sup _{0 \leq t \leq T}\left\|\left[A(t)-A_{n}(t)\right] A^{-1}(t) x\right\|\right\}=0, \quad x \in X,
$$

$A(t)$ satisfies (3), and the condition of uniform boundedness of the evolution operators corresponding to equation (14) is satisfied, i.e.

$$
\left\|U_{n}(t, s)\right\| \leq M
$$


( $M$ does not depend on $n, t$ and $s$ ), then we will say that the operator $A(t)$ is stably approximated by the operators $A_{n}(t)$.

THEOREM 1. Suppose that the operator $A(t)$ is strongly continuously differentiable on $D(A)$ for $t \in[0, T]$, has a bounded inverse and is stably approximated by operators $A_{n}(t)$ such that:

$1^{\circ}$ each $A_{n}(t)$ is strongly continuously differentiable for $t \in[0, T]$ and has a bounded inverse such that $\left\|A_{n}^{-1}(t)\right\| \leq C$ ( $C$ does not depend on $n$ and $t$ ),

$2^{\circ}$ the sequence $\left\{A_{n}^{\prime}(t) A_{n}^{-1}(t)\right\}$ converges strongly and uniformly on $[0, T]$ to a bounded operator $B(t)$.

If the evolution operators $U_{n}(t, s)$ converge, as $n \rightarrow \infty$, strongly and uniformly in $t$ and $s$ to an operator $U(t, s)$, then the Cauchy problem for equation (8) is uniformly correct and $U(t, s)$ is the evolution operator corresponding to it.

P r o of. Consider the equation

$$
\frac{d y}{d t}=A_{n}(t) y+A_{n}^{\prime}(t) A_{n}^{-1}(t) y .
$$

It follows from the continuous differentiability of $A_{n}(t)$ that the operator $A_{n}^{\prime}(t) A_{n}^{-1}(t)$ is strongly continuous (cf. [7, Lemma 1.5]). Denote by $V_{n}(t, s)$ the evolution operator corresponding to equation (17). According to Remark 2.1 in [7, p. 192] the operators $V_{n}(t, s)$ converge strongly and uniformly relative to $t$ and $s$ to a limit which we shall denote by $V(t, s)$. The operator $V(t, s)$ is strongly continuous for $t$ and $s$ in $\Delta_{T}$.

We make the substitution $A_{n}^{-1}(t) y(t, s)=x(t, s)$ in (17). Then

$$
\begin{aligned}
\frac{d x}{d t} & =A_{n}^{-1}(t) \frac{d y}{d t}-A_{n}^{-1}(t) A_{n}^{\prime}(t) A_{n}^{-1}(t) y \\
& =A_{n}^{-1}(t) A_{n}(t) y=A_{n}(t) A_{n}^{-1}(t) y=A_{n}(t) x .
\end{aligned}
$$

In view of the uniqueness of the solution of equation (14) we have

$$
A_{n}^{-1}(t) y(t, s)=x(t, s)=U_{n}(t, s) x(s, s),
$$

so that

$$
y(t, s)=A_{n}(t) U_{n}(t, s) x(s, s)=A_{n}(t) U_{n}(t, s) A_{n}^{-1}(s) y(s, s)
$$

or, in another form,

$$
V_{n}(t, s)=A_{n}(t) U_{n}(t, s) A_{n}^{-1}(s) .
$$

From (15) and the assumption $\left\|A_{n}^{-1}(t)\right\| \leq C$ we obtain

$$
\begin{aligned}
\left\|\left[A_{n}^{-1}(t)-A^{-1}(t)\right] x\right\| & \leq\left\|A_{n}^{-1}(t)\right\| \cdot\left\|\left[I-A_{n}(t) A^{-1}(t)\right] x\right\| \\
& \leq C\left\|\left[A(t)-A_{n}(t)\right] A^{-1}(t) x\right\| \rightarrow 0
\end{aligned}
$$


for each $x \in X$ uniformly in $t \in[0, T]$ as $n \rightarrow \infty$. On the other hand, by (18) we get

$$
A_{n}^{-1}(t) V_{n}(t, s) x=U_{n}(t, s) A_{n}^{-1}(s) x \quad \text { for } x \in X .
$$

Since the $U_{n}(t, s)$ converge to $U(t, s)$ and $V_{n}(t, s)$ converge to $V(t, s)$ and $A_{n}^{-1}(t)$ converge strongly and uniformly to $A^{-1}(t)$, we have, from (19),

$$
A^{-1}(t) V(t, s) x=U(t, s) A^{-1}(s) x \quad \text { for } x \in X,
$$

so that

$$
V(t, s)=A(t) U(t, s) A^{-1}(s) .
$$

Let $x_{0} \in D(A)$ and $A(s) x_{0}=y_{s}$. We have

$$
\begin{aligned}
d U_{n}(t, s) x_{0} / d t & =A_{n}(t) U_{n}(t, s) x_{0}=A_{n}(t) A_{n}^{-1}(t) V_{n}(t, s) A_{n}(s) A^{-1}(s) y_{s} \\
& =V_{n}(t, s) A_{n}(s) A^{-1}(s) y_{s} .
\end{aligned}
$$

In view of (15) the operators $A_{n}(s) A^{-1}(s)$ tend strongly, and uniformly in $s \in[0, T]$, to the identity operator, so that the derivatives $d U_{n}(t, s) x_{0} / d t$ converge uniformly in $t$ to the function $V(t, s) y_{s}=A(t) U(t, s) x_{0}$. Since the differentiation operator is closed we have

$$
\frac{d U(t, s) x_{0}}{d t}=A(t) U(t, s) x_{0},
$$

i.e. the function $x(t, s):=U(t, s) x_{0}$ is a solution of equation (8) on $[s, T]$. Since $U_{n}(s, s)=I$ for each $n \in \mathbb{N}$, we have

$$
x(s, s)=U(s, s) x_{0}=\lim _{n \rightarrow \infty} U_{n}(s, s) x_{0}=x_{0} .
$$

This means that the function $x=x(t, s)$ is a solution of the Cauchy problem (8), (9).

The uniqueness of solution of the problem (8), (9) follows from Lemma 3.1 in [7, p. 199], because each solution of this problem is given by

$$
x(t, s)=\lim _{n \rightarrow \infty} U_{n}(t, s) x_{0} .
$$

Properties $2^{\circ}$ and $3^{\circ}$ of Definition 2 follow from the boundedness and strong continuity of the operators $U(t, s)$ and $V(t, s),(t, s) \in \Delta_{T}$. Theorem 1 is proved.

Remark 1. Theorem 1 and its proof are a slight modification of Theorem 3.6 of the monograph [7, pp. 200-201]. This modification consists in omitting the assumption "the operators $A_{n}(t)$ for each $t \in[0, T]$ commute with $A(t)$ on $D(A)$ ". 
3. Fractional powers of operators. In this section we shall need the following assumptions:

$\left(\mathrm{Z}_{2}\right) \quad$ For each $t \in[0, T], A(t)$ is the infinitesimal generator of a strongly continuous cosine family $\left\{C_{t}(\xi): \xi \in \mathbb{R}\right\}$ of bounded linear operators from $X$ into itself.

$\left(\mathrm{Z}_{3}\right) \quad$ For each $t \in[0, T]$ there exists a linear operator $B(t): X \rightarrow X$ such that $B^{2}(t)=A(t)$, the domain $D(B(t)):=D(B)$ is independent of $t$ and 0 belongs to the resolvent set of $B(t)$.

$\left(\mathrm{Z}_{4}\right) \quad$ For each $x \in D(B)$ the mapping $t \rightarrow B(t) x$ is of class $C^{1}$ in $[0, T]$.

R e mark 2. In $\left(\mathrm{Z}_{3}\right)$ the essential assumption is the condition "the domain $D(B(t))=D(B)$ is independent of $t$ ". The existence of the operator $B(t)$ for each $t \in[0, T]$ may be obtained by translation of $A(t)$ without loss of generality (cf. [10]).

Let $A(t), t \in[0, T]$, satisfy $\left(\mathrm{Z}_{1}\right)-\left(\mathrm{Z}_{3}\right)$. Then the resolvent

$$
R(\lambda ; A(t)):=(\lambda-A(t))^{-1}
$$

is defined for $\lambda>0$ and satisfies the inequality

$$
\|R(\lambda ; A(t))\| \leq \frac{M}{\lambda}, \quad \lambda>0, t \in[0, T],
$$

where $M \geq 1$ is a constant independent of $\lambda$ and $t$ (cf. [2] or [4, p. 61]).

We define the operators

$$
A_{n}(t):=n A(t) R(n ; A(t)), \quad t \in[0, T], n \in \mathbb{N} .
$$

(see [7, p. 204]). Obviously, each $A_{n}(t)$ is bounded. It is proved in [7, pp. 204-205] that if $A(t)$ is strongly continuous on $D(A)$, then the operators $A_{n}(t)$ satisfy the condition (15).

By the identity (cf. [7, p. 205])

$$
R\left(\lambda ; A_{n}(t)\right)=\frac{1}{\lambda+n} I+\frac{n^{2}}{(n+\lambda)^{2}} R\left(\frac{n \lambda}{n+\lambda} ; A(t)\right)
$$

and inequality (23) we get

$$
\left\|R\left(\lambda ; A_{n}(t)\right)\right\| \leq \frac{M}{\lambda}, \quad \lambda>0, t \in[0, T],
$$

where $M$ is the constant from (23).

From (26) we deduce that it is possible to define positive fractional powers $\left(-A_{n}(t)\right)^{\alpha}(\alpha>0)$ of $-A_{n}(t)$, for each $n \in \mathbb{N}$ and $t \in[0, T]$, by

$$
\left(-A_{n}(t)\right)^{\alpha}:=\frac{\sin \alpha \pi}{\pi} \int_{0}^{\infty} \lambda^{\alpha-1} R\left(\lambda ; A_{n}(t)\right)\left(-A_{n}(t)\right) d \lambda
$$


(cf. $[4$, p. 51], [7, p. 112] or [12, Chapter IX, 11]). In formula (27) the branch of $\lambda^{\alpha-1}$ is that which is real when $\lambda \geq 0$. We let

$$
B_{n}(t):=\left[A_{n}(t)\right]^{1 / 2}:=i\left[-A_{n}(t)\right]^{1 / 2} .
$$

It is proved in [4, p. 59] that if $\mu^{2} \in \varrho\left(-A_{n}(t)\right)$ then $\mu \in \varrho\left(B_{n}(t)\right)$ and

$$
R\left(\mu ; B_{n}(t)\right)=\frac{1}{\pi} \int_{0}^{\infty} \frac{\lambda^{1 / 2}}{\lambda+\mu^{2}} R\left(\lambda ; A_{n}(t)\right) d \lambda .
$$

From (29) by the estimate (26) we get

$$
\left\|R\left(\mu ; B_{n}(t)\right)\right\| \leq \frac{M}{|\mu|}, \quad \mu \neq 0, t \in[0, T],
$$

where $M$ is the constant of $(26)$.

Lemma 2. Assume $\left(\mathrm{Z}_{1}\right)$ and $\left(\mathrm{Z}_{2}\right)$. If the family of generators $\{A(t)\}$, $t \in[0, T]$, is stable with constants $M \geq 1$ and $\omega=0$ (called the stability constants), i.e., the following conditions are satisfied:

$$
\begin{aligned}
\|\left(\lambda_{k}-A\left(t_{k}\right)\right)^{-1}\left(\lambda_{k-1}-A\left(t_{k-1}\right)\right)^{-1} \ldots( & \left.\lambda_{1}-A\left(t_{1}\right)\right)^{-1} \| \\
& \leq M \prod_{j=1}^{k} \lambda_{j}^{-1}, \quad \lambda_{j}>0,
\end{aligned}
$$

and

$$
\begin{aligned}
&\left\|\left(\lambda_{1}-A\left(t_{1}\right)\right)^{-1}\left(\lambda_{2}-A\left(t_{2}\right)\right)^{-1} \ldots\left(\lambda_{k}-A\left(t_{k}\right)\right)^{-1}\right\| \\
& \leq M \prod_{j=1}^{k}\left(-\lambda_{j}\right)^{-1}, \quad \lambda_{j}<0,
\end{aligned}
$$

then the family $\left\{B_{n}(t)\right\}, t \in[0, T], n \in \mathbb{N}$, is uniformly twice-stable, i.e., the following conditions are satisfied:

$$
\left\|\left(\mu-B_{n}\left(t_{k}\right)\right)^{-1}\left(\mu-B_{n}\left(t_{k-1}\right)\right)^{-1} \ldots\left(\mu-B_{n}\left(t_{1}\right)\right)^{-1}\right\| \leq M|\mu|^{-k}
$$

and

$$
\left\|\left(\mu-B_{n}\left(t_{1}\right)\right)^{-1}\left(\mu-B_{n}\left(t_{2}\right)\right)^{-1} \ldots\left(\mu-B_{n}\left(t_{k}\right)\right)^{-1}\right\| \leq M|\mu|^{-k},
$$

for $\mu \neq 0$ and any finite sequence $0 \leq t_{1} \leq \ldots \leq t_{k} \leq T, k, n \in \mathbb{N}$.

Proof. In [1, Lemma 1] we proved that if a family $\{A(t)\}, t \in[0, T]$, is stable with stability constants $M$ and $\omega$, then the family $\left\{A_{n}(t)\right\}, t \in[0, T]$, $n \in \mathbb{N}$, where $A_{n}(t)$ is defined by (24), is uniformly stable with stability constants $M$ and $2 \omega$. Since by assumption $\omega=0$, the family $\left\{A_{n}(t)\right\}, t \in$ $[0, T], n \in \mathbb{N}$, is uniformly stable with constants $M$ and $\omega=0$. 
As remarked above, if $\lambda \in \varrho\left(-A_{n}(t)\right)$ and $\lambda>0$ then $\mu \in \varrho\left(B_{n}(t)\right)$, where $\mu^{2}=\lambda$. From this, by (29), we have

$$
\begin{aligned}
\left(\mu-B_{n}\left(t_{j}\right)\right)^{-1}= & \frac{1}{\pi} \int_{0}^{\infty} \frac{\lambda_{j}^{1 / 2}}{\lambda_{j}+\mu^{2}}\left(\lambda_{j}-A_{n}\left(t_{j}\right)\right)^{-1} d \lambda_{j}, \\
& j=1, \ldots, k, \lambda_{j}>0,0 \leq t_{1} \leq t_{2} \leq \ldots \leq t_{k} \leq T .
\end{aligned}
$$

Hence

$$
\begin{aligned}
(\mu & \left.-B_{n}\left(t_{k}\right)\right)^{-1}\left(\mu-B_{n}\left(t_{k-1}\right)\right)^{-1} \ldots\left(\mu-B_{n}\left(t_{1}\right)\right)^{-1} \\
& =\pi^{-k} \int_{0}^{\infty} \ldots \int_{0}^{\infty} \prod_{j=1}^{k} \frac{\lambda_{j}^{1 / 2}}{\lambda_{j}+\mu^{2}}\left(\lambda_{k}-A_{n}\left(t_{k}\right)\right)^{-1} \ldots\left(\lambda_{1}-A_{n}\left(t_{1}\right)\right)^{-1} d \lambda_{k} \ldots d \lambda_{1} .
\end{aligned}
$$

From this, by the uniform stability of the family $\left\{A_{n}(t)\right\}, t \in[0, T], n \in \mathbb{N}$, we obtain

$$
\begin{aligned}
\|\left(\mu-B_{n}\left(t_{k}\right)\right)^{-1}\left(\mu-B_{n}\left(t_{k-1}\right)\right)^{-1} & \ldots\left(\mu-B_{n}\left(t_{1}\right)\right)^{-1} \| \\
\leq & M \pi^{-k} \int_{0}^{\infty} \ldots \int_{0}^{\infty} \prod_{j=1}^{k} \frac{\lambda_{j}^{-1 / 2} d \lambda_{j}}{\lambda_{j}+\mu^{2}} \\
& =M \prod_{j=1}^{k}\left(\frac{1}{\pi} \int_{0}^{\infty} \frac{\lambda_{j}^{-1 / 2} d \lambda_{j}}{\lambda_{j}+\mu^{2}}\right)=M|\mu|^{-k} .
\end{aligned}
$$

Inequality (33) is proved. The proof of (34) is similar and so we omit it.

Lemma 3. Let $A(t), t \in[0, T]$, be the operator defined in the introduction. If $A(t)$ satisfies inequalities (3) and (23), then for each $n \in \mathbb{N}$ and any $t \in[0, T]$ the operator $B_{n}(t)$ defined by (28) is invertible and

$$
\left\|B_{n}^{-1}(t)\right\| \leq C
$$

where $C$ does not depend on $n$ and $t$.

P r o o f. From (28) we get

$$
B_{n}^{-1}(t)=\left[A_{n}(t)\right]^{-1 / 2}=\frac{1}{i}\left[-A_{n}(t)\right]^{-1 / 2} .
$$

It follows that (see $[7$, p. 112])

$$
B_{n}^{-1}(t)=\frac{1}{\pi i} \int_{0}^{\infty} \lambda^{-1 / 2} R\left(\lambda ; A_{n}(t)\right) d \lambda,
$$

where $A_{n}(t)$ is defined by (24). 
In order to prove the existence of $B_{n}^{-1}(t)$ it suffices to show the convergence of the integral in (37). We have

$$
\begin{aligned}
\int_{0}^{\infty} \lambda^{-1 / 2} R\left(\lambda ; A_{n}(t)\right) d \lambda \\
\quad=\int_{0}^{1} \lambda^{-1 / 2} R\left(\lambda ; A_{n}(t)\right) d \lambda+\int_{1}^{\infty} \lambda^{-1 / 2} R\left(\lambda ; A_{n}(t)\right) d \lambda \\
=\int_{0}^{1} \lambda^{-1 / 2} A_{n}(t) R\left(\lambda ; A_{n}(t)\right) A_{n}^{-1}(t) d \lambda+\int_{1}^{\infty} \lambda^{-1 / 2} R\left(\lambda ; A_{n}(t)\right) d \lambda \\
=\int_{0}^{1} \lambda^{-1 / 2}\left[\lambda R\left(\lambda ; A_{n}(t)\right)-I\right] A_{n}^{-1}(t) d \lambda+\int_{1}^{\infty} \lambda^{-1 / 2} R\left(\lambda ; A_{n}(t)\right) d \lambda .
\end{aligned}
$$

By (24) we obtain

$$
A_{n}^{-1}=A^{-1}(t)-\frac{1}{n} I .
$$

It follows from (3) that $\left\|A^{-1}(t)\right\| \leq C_{1}$ for $t \in[0, T]$, and so $\left\|A_{n}^{-1}(t)\right\| \leq$ $C_{1}+1$. From this and from (23) we get

$\left\|\int_{0}^{1} \lambda^{-1 / 2} R\left(\lambda ; A_{n}(t)\right) d \lambda\right\| \leq(M+1)\left(C_{1}+1\right) \int_{0}^{1} \lambda^{-1 / 2} d \lambda=2(M+1)\left(C_{1}+1\right)$

and

$$
\left\|\int_{1}^{\infty} \lambda^{-1 / 2} R\left(\lambda ; A_{n}(t)\right) d \lambda\right\| \leq M \int_{1}^{\infty} \lambda^{-3 / 2} d \lambda=2 M,
$$

which proves the existence of $B_{n}^{-1}(t)$, and also the estimate (36) with $C:=$ $\frac{2}{\pi}\left[(M+1)\left(C_{1}+1\right)+M\right]$.

LEMma 4. Under the assumptions of Lemma 3, if the mapping $[0, T] \ni$ $t \rightarrow A(t) x$ is continuous for $x \in D(A)$ then the sequence $\left\{B_{n}(t)\right\}$ is strongly and uniformly convergent to the operator $B(t)$ on the domain $D(A)$, where $B(t)$ is taken from assumption $\left(\mathrm{Z}_{3}\right)$.

Proof. From the definition of $B(t)$ and from inequality (23) it follows that for $x \in D(A)$ we have

$$
B(t) x=[A(t)]^{1 / 2} x=\frac{i}{\pi} \int_{0}^{\infty} \lambda^{-1 / 2} R(\lambda ; A(t))(-A(t) x) d \lambda, \quad t \in[0, T] .
$$

Combining (27) with (28) we obtain

$$
B_{n}(t) x=\frac{i}{\pi} \int_{0}^{\infty} \lambda^{-1 / 2} R\left(\lambda ; A_{n}(t)\right)\left(-A_{n}(t) x\right) d \lambda, \quad x \in X, t \in[0, T] .
$$


Fix $x \in D(A)$. In this case, similarly to Lemma 3 , one may prove that the improper integral in (39), as well in (38), is convergent absolutely and uniformly relative to $t$ and $n$.

By the definition of $A_{n}(t)$ and by the strong continuity of the mapping $t \rightarrow A(t)$, we have, for $x \in D(A)$ and $t \in[0, T]$,

$$
\begin{aligned}
\left\|\left[A_{n}(t)-A(t)\right] A^{-1}(t) x\right\| & =\left\|[n R(n ; A(t)) A(t)-A(t)] A^{-1}(t) x\right\| \\
& =\|R(n ; A(t)) A(t) x\| \\
& \leq \frac{M}{n} \max _{0 \leq t \leq T}\|A(t) x\| \rightarrow 0 \quad \text { as } n \rightarrow \infty .
\end{aligned}
$$

The norms of the operators $n R(n ; A(t))$ are bounded by $M$, so that in view of the Banach-Steinhaus theorem the operators $A_{n}(t) A^{-1}(t)$ tend to the identity operator strongly and uniformly in $t \in[0, T]$. Therefore, the operators $A_{n}(t)$ tend strongly and uniformly on $D(A)$ to the operator $A(t)$. Letting $n \rightarrow \infty$ in (39) we get

$$
\lim _{n \rightarrow \infty} B_{n}(t) x=\frac{i}{\pi} \int_{0}^{\infty} \lambda^{-1 / 2} R(\lambda ; A(t))(-A(t)) x d \lambda=B(t) x, \quad x \in D(A) .
$$

Hence,

$$
B_{n}(t) x \rightarrow B(t) x \quad \text { as } n \rightarrow \infty \quad \text { for } x \in D(A),
$$

uniformly in $t \in[0, T]$.

Formula (38) defines the operator $B(t)$ on $D(A)$.

The entire operator $B(t)$ may be obtained by closure from its restriction to $D(A)$ (cf. [7, p. 114]). As in the proof of Lemma 3, we may show that the operator $B(t)$ is invertible for each $t \in[0, T]$ and

$$
\left\|B^{-1}(t)\right\| \leq C
$$

where $C$ is the same as in (36).

The domain $D(B(t))=D(B)$, by assumption $\left(\mathrm{Z}_{3}\right)$, is dense in $X$ and contains $D(A)$.

In the sequel we shall use a generalized convergence of sequences of operators (see [5, Section IV. 2]). We only use a sufficient condition for the generalized convergence (see [5, Theorem 2.29, p. 207]), and we make the following definition.

Definition 5. Let $T_{n}(t), T(t) \in \mathcal{C}(X)$ for $t \in[a, b]$. Consider a second Banach space $Y$ and operators $U_{n}(t), U(t), V_{n}(t), V(t) \in B(Y, X)$ such that $U_{n}(t), U(t)$ map $Y$ one-to-one onto $D\left(T_{n}(t)\right), D(T(t))$, respectively, and $T_{n}(t) U_{n}(t)=V_{n}(t), T(t) U(t)=V(t)$. If, for each $x \in Y, U_{n}(t) x \rightarrow U(t) x$ and $V_{n}(t) x \rightarrow V(t) x$ as $n \rightarrow \infty$ for $t \in[a, b]$, then we shall say that the sequence $\left\{T_{n}(t)\right\}$ converges strongly in $[a, b]$ to the operator $T(t)$ in the generalized sense. 
Lemma 5. Let assumptions $\left(\mathrm{Z}_{1}\right)-\left(\mathrm{Z}_{4}\right)$ hold. If the mapping $[0, T] \ni t \rightarrow$ $A(t) x, x \in D(A)$, is continuous and inequality (3) is satisfied, then the operators $B_{n}(t) B^{-1}(s)$ are uniformly bounded in the square $K_{T}:=[0, T] \times$ $[0, T]$ and

$$
B_{n}(t) B^{-1}(s) x \rightarrow B(t) B^{-1}(s) x \quad \text { for } x \in X,
$$

uniformly in $K_{T}$.

Proof. By (3) and the strong continuity of the operator $A(t)$ we get the uniform boundedness of the operator $A(t) A^{-1}(s)$ in $K_{T}$. From this, by (38), we obtain the uniform boundedness of $B(t) A^{-1}(s)$ in $K_{T}$. Analogously, from (39) we get the uniform boundedness of the sequence $\left\{B_{n}(t) A^{-1}(s)\right\}$ in $K_{T}$. Next, from (40), it follows that

$$
B_{n}(t) A^{-1}(s) x \rightarrow B(t) A^{-1}(s) x \quad \text { as } n \rightarrow \infty \quad \text { for } x \in X,
$$

uniformly in the square $K_{T}$.

From (42), by Definition 5, we deduce that the sequence $\left\{B_{n}(t)\right\}$, where the $B_{n}(t)$ are defined in the subspace $D(A)$, converges strongly and uniformly to the operator $B(t)$ on $D(A)$ for $t \in[0, T]$ in the generalized sense.

Consider now the sequence $\left\{B_{n}(t) B^{-1}(t)\right\}$, defined on $D(B)$, where $D(B)$ is taken from assumption $\left(\mathrm{Z}_{3}\right)$. We proceed to show that

$$
B_{n}(t) B^{-1}(s) \rightarrow B(t) B^{-1}(s)
$$

uniformly in $K_{T}$, in the sense of Definition 5 . Indeed, we take

$$
Y:=X, \quad U_{n}(s):=B^{-1}(s), \quad U(s):=B^{-1}(s) \text { for } s \in[0, T] .
$$

It follows that $V_{n}(t, s)=B_{n}(t) A^{-1}(s)$ and $V(t, s)=B(t) A^{-1}(s),(t, s) \in$ $K_{T}$. From this, by (42), we obtain (43).

On the other hand, by assumption $\left(\mathrm{Z}_{3}\right)$, the operator $B(t) B^{-1}(s): X \rightarrow$ $X$ is bounded uniformly in $K_{T}$. Therefore, by Theorem 2.23 in [5, p. 206], the operators $B_{n}(t) B^{-1}(s)$ are uniformly bounded in $K_{T}$ for sufficiently large $n$ and

$$
\left\|B_{n}(t) B^{-1}(s) x-B(t) B^{-1}(s) x\right\| \rightarrow 0, \quad \text { for } x \in D(B),
$$

uniformly in $K_{T}$. Using the Banach-Steinhaus theorem we obtain the assertion of Lemma 5.

LEMma 6. If assumption $\left(\mathrm{Z}_{1}\right)$ holds, the mapping $[0, T] \ni t \rightarrow A(t) x$, $x \in D(A)$, is of class $C^{1}$ and inequality (23) is satisfied, then the mapping $[0, T] \ni t \rightarrow A_{n}(t) x, x \in D(A)$, is of class $C^{1}$, where $A_{n}(t)$ is given by (24), and: 


$$
\begin{aligned}
A_{n}^{\prime}(t) A_{n}^{-1}(t) x & \rightarrow A^{\prime}(t) A^{-1}(t) x & & \text { as } n \rightarrow \infty, \text { for } x \in X, \\
A_{n}^{\prime}(t) A^{-1}(s) x & \rightarrow A^{\prime}(t) A^{-1}(s) x & & \text { as } n \rightarrow \infty, \text { for } x \in X, \\
A_{n}^{\prime}(t) x & \rightarrow A^{\prime}(t) x & & \text { as } n \rightarrow \infty, \text { for } x \in D(A),
\end{aligned}
$$

uniformly in $t \in[0, T]$ or $(t, s) \in K_{T}$, respectively.

Proof. Let us remark that the operator $A_{n}(t)$ may be written in the form

$$
A_{n}(t)=n^{2} R(n ; A(t))-n I .
$$

Formula (47) implies the existence of $A_{n}^{\prime}(t)$ in the strong sense, and

$$
A_{n}^{\prime}(t)=n^{2} R(n ; A(t)) A^{\prime}(t) R(n ; A(t)),
$$

and so the mapping $[0, T] \ni t \rightarrow A_{n}^{\prime}(t) x, x \in D(A)$, is continuous.

By (48) and (24) we get

$$
A_{n}^{\prime}(t) A_{n}^{-1}(t) x=n R(n ; A(t))\left[A^{\prime}(t) A^{-1}(t)\right] x .
$$

Since the bounded operators $n R(n ; A(t))$ tend to the identity operator strongly and uniformly in $t \in[0, T]$, we have (44). Further,

$$
A_{n}^{\prime}(t) A^{-1}(s) x=\left[A_{n}^{\prime}(t) A_{n}^{-1}(t)\right]\left[A_{n}(t) A^{-1}(t)\right]\left[A(t) A^{-1}(s) x\right] .
$$

Because the operators $A_{n}^{\prime}(t) A^{-1}(t)$ and $A_{n}(t) A^{-1}(t)$ are bounded and tend to $A^{\prime}(t) A^{-1}(t)$ and the identity operator, respectively, by Lemmas 3.7 and 3.8 in [5, p. 151] we obtain (45); (46) follows immediately from (45).

Lemma 7. Assume $\left(\mathrm{Z}_{1}\right)-\left(\mathrm{Z}_{4}\right)$. If the operator $A(t)$ for $t \in[0, T]$ satisfies inequality (23) and the mapping

$$
[0, T] \ni t \rightarrow A(t) x, \quad x \in D(A),
$$

is of class $C^{1}$, then for every $n \in \mathbb{N}$ and $x \in D(A)$ the mapping

$$
[0, T] \ni t \rightarrow B_{n}(t) x
$$

is of class $C^{1}$ and

$$
B_{n}^{\prime}(t) B^{-1}(s) x \rightarrow B^{\prime}(t) B^{-1}(s) x, \quad x \in X,
$$

uniformly in the square $K_{T}$.

Proof. From (24) we see that if the mapping (49) is of class $C^{1}$, then so is

$$
[0, T] \ni t \rightarrow A_{n}(t) x, \quad x \in D(A), n \in \mathbb{N} .
$$

From (39) we get

$$
B_{n}(t) x=\frac{i}{\pi} \int_{0}^{\infty} \lambda^{-1 / 2}\left[I-\lambda R\left(\lambda ; A_{n}(t)\right)\right] d \lambda .
$$


Fix $x \in D(A)$. We let

$$
\begin{aligned}
I(t) & :=\frac{i}{\pi} \int_{0}^{\infty} \frac{d}{d t}\left\{\lambda^{-1 / 2}\left[I-\lambda R\left(\lambda ; A_{n}(t)\right)\right]\right\} x d \lambda \\
& =\frac{i}{\pi} \int_{0}^{\infty} \lambda^{1 / 2} R\left(\lambda ; A_{n}(t)\right)\left(-A_{n}^{\prime}(t)\right) R\left(\lambda ; A_{n}(t)\right) x d \lambda=I_{1}(t)+I_{2}(t),
\end{aligned}
$$

where

$$
I_{1}(t):=\frac{i}{\pi} \int_{0}^{1} \lambda^{1 / 2} R\left(\lambda ; A_{n}(t)\right)\left(-A_{n}^{\prime}(t)\right) R\left(\lambda ; A_{n}(t)\right) x d \lambda
$$

and

$$
I_{2}(t):=\frac{i}{\pi} \int_{1}^{\infty} \lambda^{1 / 2} R\left(\lambda ; A_{n}(t)\right)\left(-A_{n}^{\prime}(t)\right) R\left(\lambda ; A_{n}(t)\right) x d \lambda .
$$

From this we get

$$
\begin{aligned}
\left\|I_{1}(t)\right\| \leq & \frac{1}{\pi} \int_{0}^{1} \lambda^{-1 / 2}\left\|\lambda R\left(\lambda ; A_{n}(t)\right)\right\| \\
& \times\left\|A_{n}^{\prime}(t) A_{n}^{-1}(t)\right\| \cdot\left\|A_{n}(t) R\left(\lambda ; A_{n}(t)\right)\right\| \cdot\|x\| d \lambda \\
\leq & \frac{1}{\pi} M C(M+1)\|x\| \int_{0}^{1} \lambda^{-1 / 2} d \lambda=\frac{2}{\pi} M(M+1) C\|x\|
\end{aligned}
$$

and

$$
\begin{aligned}
\left\|I_{2}(t)\right\| \leq & \frac{1}{\pi} \int_{1}^{\infty} \lambda^{-3 / 2}\left\|\lambda R\left(\lambda ; A_{n}(t)\right)\right\|^{2} \| \\
& \times\left\|A_{n}^{\prime}(t) A_{n}^{-1}(t)\right\| \cdot\left\|A_{n}(t) A^{-1}(t)\right\| \cdot\|A(t) x\| d \lambda \\
\leq & \frac{2}{\pi} C M^{2} C_{1} \sup _{0 \leq t \leq T}\|A(t) x\|,
\end{aligned}
$$

where

$$
\left\|A_{n}^{\prime}(t) A_{n}^{-1}(t)\right\| \leq C, \quad\left\|A_{n}(t) A^{-1}(t)\right\| \leq C_{1} \quad \text { and } \quad\left\|\lambda R\left(\lambda ; A_{n}(t)\right)\right\| \leq M .
$$

From (52) and (53) it follows that for $x \in D(A)$ and $n \in \mathbb{N}$ we have

$$
B_{n}^{\prime}(t) x=\frac{i}{\pi} \int_{0}^{\infty} \lambda^{1 / 2} R\left(\lambda ; A_{n}(t)\right)\left(-A_{n}^{\prime}(t)\right) R\left(\lambda ; A_{n}(t)\right) x d \lambda .
$$

Using (52) and (53) once more, and in view of the well-known theorem on the passing to the limit under the improper integral in (54), for $x \in D(A)$ 
we obtain

$$
B^{\prime}(t) x=\frac{i}{\pi} \int_{0}^{\infty} \lambda^{1 / 2} R(\lambda ; A(t))\left(-A^{\prime}(t)\right) R(\lambda ; A(t)) x d \lambda .
$$

We conclude from (55) that

$$
B_{n}^{\prime}(t) x \rightarrow B^{\prime}(t) x \quad \text { as } n \rightarrow \infty \text { for } x \in D(A) .
$$

The same reasoning as in the proof of Lemma 5 shows that (50) holds. Lemma 7 is proved.

COROLLARY 1. Under the assumptions of Lemma 7, formula (50) implies that there exists a constant $L>0$ such that

$$
\left\|B_{n}^{\prime}(t) B^{-1}(s)\right\| \leq L
$$

where $L$ does not depend on $(t, s) \in K_{T}$ and on $n \in \mathbb{N}$.

4. Second order initial value problem. Similarly to Section 2, we now consider the homogeneous differential equation corresponding to problem (7), i.e.

$$
\frac{d^{2} u}{d t^{2}}=A(t) u, \quad t \in[0, T]
$$

where $A(t)$ is the operator defined in the introduction and satisfying assumption $\left(\mathrm{Z}_{1}\right)$ and inequality (23). It follows that, for each $t \in[0, T]$, there is a linear operator $B(t): X \rightarrow X$ such that

$$
B^{2}(t) x=A(t) x \quad \text { for } x \in D(A), t \in[0, T] .
$$

We assume that the operator $B(t)$ satisfies $\left(\mathrm{Z}_{3}\right)$ and $\left(\mathrm{Z}_{4}\right)$.

Definition 6 (cf. [6]). A family $S$ of bounded operators $S(t, s): X \rightarrow$ $X, t, s \in[0, T]$, is said to be the fundamental solution for equation (57) if:

$\left(\mathrm{D}_{1}\right) \quad$ For each $x \in X$ the mapping $K_{T} \ni(t, s) \rightarrow S(t, s) x \in X$ is of class $C^{1}$ and

(a) for each $t \in[0, T], S(t, t)=0$,

(b) for all $t, s \in[0, T]$ and each $x \in X$,

$$
\left.\frac{\partial}{\partial t} S(t, s)\right|_{t=s} x=x,\left.\quad \frac{\partial}{\partial s} S(t, s)\right|_{t=s} x=-x .
$$

$\left(\mathrm{D}_{2}\right) \quad$ For all $t, s \in[0, T]$, if $x \in D(B)$ then $S(t, s) x \in D(A)$ and the mapping $[0, T] \ni t \rightarrow S(t, s) x \in X$ is of class $C^{2}$; moreover,

$$
\begin{aligned}
\frac{\partial^{2}}{\partial t^{2}} S(t, s) x & =A(t) S(t, s) x, \\
\left.\frac{\partial^{2}}{\partial s \partial t} S(t, s)\right|_{t=s} x & =0,
\end{aligned}
$$


while for $x \in D(A)$ the mapping $K_{T} \ni(t, s) \rightarrow S(t, s) x \in X$ is of class $C^{2}$ and

$$
\frac{\partial^{2}}{\partial s^{2}} S(t, s) x=S(t, s) A(s) x .
$$

$\left(\mathrm{D}_{3}\right) \quad$ For all $t, s \in[0, T]$, if $x \in D(A)$ then $\frac{\partial}{\partial s} S(t, s) x \in D(A)$, the derivatives $\frac{\partial^{3}}{\partial t^{2} \partial s} S(t, s) x, \frac{\partial^{3}}{\partial s^{2} \partial t} S(t, s) x$ exist and

$$
\begin{aligned}
& \frac{\partial^{3}}{\partial t^{2} \partial s} S(t, s) x=A(t) \frac{\partial}{\partial s} S(t, s) x, \\
& \frac{\partial^{3}}{\partial s^{2} \partial t} S(t, s) x=\frac{\partial}{\partial t} S(t, s) A(s) x,
\end{aligned}
$$

and the mapping $K_{T} \ni(t, s) \rightarrow A(t) \frac{\partial}{\partial s} S(t, s) x$ is continuous.

$\left(\mathrm{D}_{4}\right) \quad$ For all $t, s, r \in[0, T]$,

$$
S(t, s)=S(t, r) \frac{\partial}{\partial r} S(r, s)-\frac{\partial}{\partial r} S(t, r) S(r, s) .
$$

Under assumptions $\left(\mathrm{Z}_{1}\right)-\left(\mathrm{Z}_{4}\right)$ we denote by $Y$ the linear space $D(B)$ with the norm $\|\cdot\|_{Y}$ given by

$$
\|y\|_{Y}:=\|y\|+\|B(0) y\|, \quad y \in D(B) .
$$

Let $\mathcal{A}(t), t \in[0, T]$, be a linear operator such that $\mathcal{A}(t): Y \times X \rightarrow Y \times X$, where

$$
\mathcal{A}(t):=\left[\begin{array}{cc}
0 & I \\
A(t) & 0
\end{array}\right], \quad t \in[0, T],
$$

with domain $D(A) \times D(B)$. We define the sequence $\left\{\mathcal{A}_{n}(t)\right\}$ of linear operators acting from $Y \times X$ into itself by

$$
\mathcal{A}_{n}(t):=\left[\begin{array}{cc}
0 & I \\
A_{n}(t) & 0
\end{array}\right] \quad \text { for } t \in[0, T], n \in \mathbb{N},
$$

where $A_{n}(t)$ is defined by formula (24).

TheOREM 2. Under assumptions $\left(\mathrm{Z}_{1}\right)$ and $\left(\mathrm{Z}_{3}\right)$, if the mapping $[0, T]$ $\ni t \rightarrow A(t) x$, for $x \in D(A)$ is of class $C^{1}$ and inequality (23) holds, then the sequence $\left\{\mathcal{A}_{n}(t)\right\}$ satisfies the following conditions:

$1^{\circ}$ for each $n \in \mathbb{N}$, the operator $\mathcal{A}_{n}(t), t \in[0, T]$, is bounded;

$2^{\circ}$ the mapping $[0, T] \ni t \rightarrow \mathcal{A}_{n}(t)$ is strongly continuously differentiable;

$3^{\circ}$ the operator $\mathcal{A}_{n}(t)$ is invertible for each $t \in[0, T]$ and

$$
\left\|\mathcal{A}_{n}^{-1}(t)\right\| \leq C \quad(C \text { does not depend on } n \in \mathbb{N} \text { and } t) ;
$$

$4^{\circ}$ the sequence $\left\{\mathcal{A}_{n}^{\prime}(t) \mathcal{A}_{n}^{-1}(t)\right\}$ is strongly and uniformly convergent to a bounded operator $\mathcal{G}(t)$;

$5^{\circ} \lim _{n \rightarrow \infty}\left\{\sup _{0 \leq t \leq T}\left\|\left[\mathcal{A}_{n}(t)-\mathcal{A}(t)\right] \mathcal{A}^{-1}(t)\left[\begin{array}{l}y \\ x\end{array}\right]\right\|\right\}=0, \quad(y, x) \in Y \times X$. 
Proof. $1^{\circ}$ Let us remark that for each $t \in[0, T]$, we have

$$
\mathcal{A}_{n}(t):=\left[\begin{array}{cc}
B_{n}^{-1}(t) & 0 \\
0 & I
\end{array}\right]\left[\begin{array}{cc}
0 & B_{n}(t) \\
B_{n}(t) & 0
\end{array}\right]\left[\begin{array}{cc}
B_{n}(t) & 0 \\
0 & I
\end{array}\right],
$$

where

$$
\begin{gathered}
{\left[\begin{array}{cc}
B_{n}^{-1}(t) & 0 \\
0 & I
\end{array}\right]: X \times X \rightarrow Y \times X, \quad\left[\begin{array}{cc}
B_{n}(t) & 0 \\
0 & I
\end{array}\right]: Y \times X \rightarrow X \times X,} \\
{\left[\begin{array}{cc}
0 & B_{n}(t) \\
B_{n}(t) & 0
\end{array}\right]: X \times X \rightarrow X \times X}
\end{gathered}
$$

(cf. [10]). It is easy to prove that each operator on the right-hand side of (63) is bounded. Indeed,

$$
\begin{aligned}
\left\|\left[\begin{array}{cc}
B_{n}^{-1}(t) & 0 \\
0 & I
\end{array}\right]\left[\begin{array}{c}
y \\
x
\end{array}\right]\right\| & =\left\|\left[\begin{array}{c}
B_{n}^{-1}(t) y \\
x
\end{array}\right]\right\| \\
& =\left\|B_{n}^{-1}(t) y\right\|+\left\|B(0) B_{n}^{-1}(t) y\right\|+\|x\| \\
& \leq \alpha\|y\|+\beta\|y\|+\|x\| \\
& \leq \gamma(\|y\|+\|x\|)=\gamma\left\|\left[\begin{array}{c}
y \\
x
\end{array}\right]\right\|,
\end{aligned}
$$

where $\gamma=\max (\alpha+\beta, 1)$ and the existence of the constants $\alpha$ and $\beta$ follows from Lemma 5. Thus

$$
\left\|\left[\begin{array}{cc}
B_{n}^{-1}(t) & 0 \\
0 & I
\end{array}\right]\right\| \leq \gamma .
$$

Further,

$$
\begin{aligned}
\left\|\left[\begin{array}{cc}
B_{n}(t) & 0 \\
0 & I
\end{array}\right]\left[\begin{array}{l}
y \\
x
\end{array}\right]\right\| & =\left\|\left[\begin{array}{c}
B_{n}(t) y \\
x
\end{array}\right]\right\|=\left\|B_{n}(t) y\right\|+\|x\| \\
& =\left\|B_{n}(t) B^{-1}(0) B(0) y\right\|+\|x\| \\
& \leq\left\|B_{n}(t) B^{-1}(0)\right\| \cdot\|B(0) y\|+\|x\| \\
& \leq a\|B(0) y\|+\|x\| \leq a(\|y\|+\|B(0) y\|)+\|x\| \\
& \leq b(\|y\|+\|B(0) y\|+\|x\|)=b\left\|\left[\begin{array}{c}
y \\
x
\end{array}\right]\right\|,
\end{aligned}
$$

where $b=\max (a, 1)$ and the existence of the constant $a$ follows from Lemma 5. Thus

$$
\left\|\left[\begin{array}{cc}
B_{n}(t) & 0 \\
0 & I
\end{array}\right]\right\| \leq b
$$

Since

$$
\left\|\left[\begin{array}{cc}
0 & B_{n}(t) \\
B_{n}(t) & 0
\end{array}\right]\right\|=2\left\|B_{n}(t)\right\|,
$$

part $1^{\circ}$ is proved. 
$2^{\circ}$ follows imediately from the assumptions.

$3^{\circ}$ From (63) we get

$$
\mathcal{A}_{n}^{-1}(t)=\left[\begin{array}{cc}
B_{n}^{-1}(t) & 0 \\
0 & I
\end{array}\right]\left[\begin{array}{cc}
0 & B_{n}^{-1}(t) \\
B_{n}^{-1}(t) & 0
\end{array}\right]\left[\begin{array}{cc}
B_{n}(t) & 0 \\
0 & I
\end{array}\right] .
$$

Hence

$$
\begin{aligned}
\left\|\mathcal{A}_{n}^{-1}(t)\right\| & \leq\left\|\left[\begin{array}{cc}
B_{n}^{-1}(t) & 0 \\
0 & I
\end{array}\right]\right\|\left\|\left[\begin{array}{cc}
0 & B_{n}^{-1}(t) \\
B_{n}^{-1}(t) & 0
\end{array}\right]\right\|\left\|\left[\begin{array}{cc}
B_{n}(t) & 0 \\
0 & I
\end{array}\right]\right\| \\
& \leq \gamma 2\left\|B_{n}^{-1}(t)\right\| b \leq 2 \alpha \gamma b:=C,
\end{aligned}
$$

where $C$ does not dependend on $t \in[0, T]$ and $n \in \mathbb{N}$.

$4^{\circ}$ An easy computation shows that

$$
\mathcal{A}_{n}^{\prime}(t) \mathcal{A}_{n}^{-1}(t)=\left[\begin{array}{cc}
0 & 0 \\
0 & A_{n}^{\prime}(t) A_{n}^{-1}(t)
\end{array}\right] .
$$

From this, by formula (44) of Lemma 6 , we get $4^{\circ}$, where the operator $\mathcal{G}(t)$ is given by

$$
\mathcal{G}(t)=\left[\begin{array}{cc}
0 & 0 \\
0 & A^{\prime}(t) A^{-1}(t)
\end{array}\right] .
$$

It is evident that $\mathcal{G}(t)$ is bounded and

$$
\|\mathcal{G}(t)\| \leq\left\|A^{\prime}(t) A^{-1}(t)\right\| .
$$

$5^{\circ}$ It is easy to check that

$$
\left\|\left[\mathcal{A}_{n}(t)-\mathcal{A}(t)\right] \mathcal{A}^{-1}(t)\left[\begin{array}{c}
y \\
x
\end{array}\right]\right\|=\left\|\left[A_{n}(t)-A(t)\right] A^{-1}(t) x\right\|, \quad x \in X, y \in Y .
$$

This and (15) imply $5^{\circ}$, and the proof is complete.

For each $t \in[0, T]$ and $n \in \mathbb{N}$ let us denote by $\mathcal{B}_{n}(t)$ the linear operator from $X \times X$ into itself given by

$$
\mathcal{B}_{n}(t)=\left[\begin{array}{cc}
0 & B_{n}(t) \\
B_{n}(t) & 0
\end{array}\right]
$$

Using Lemma 2.4 of [6] and our Lemma 2 we get

LEMMA 8. Under the assumptions of Lemma 2 , the family $\left\{\mathcal{B}_{n}(t)\right\}, t \in$ $[0, T], n \in \mathbb{N}$, is uniformly stable with stability constants $\bar{M}=2 M$ and $\bar{\omega}=0$, i.e., the following inequalities hold:

$$
\left\|\left(\lambda-\mathcal{B}_{n}\left(t_{k}\right)\right)^{-1}\left(\lambda-\mathcal{B}_{n}\left(t_{k-1}\right)\right)^{-1} \ldots\left(\lambda-\mathcal{B}_{n}\left(t_{1}\right)\right)^{-1}\right\| \leq 2 M|\lambda|^{-k}
$$

$$
\left\|\left(\lambda-\mathcal{B}_{n}\left(t_{1}\right)\right)^{-1}\left(\lambda-\mathcal{B}_{n}\left(t_{2}\right)\right)^{-1} \ldots\left(\lambda-\mathcal{B}_{n}\left(t_{k}\right)\right)^{-1}\right\| \leq 2 M|\lambda|^{-k} .
$$

TheOREM 3. Let assumptions $\left(\mathrm{Z}_{1}\right)-\left(\mathrm{Z}_{4}\right)$ hold. If the family of generators $\{A(t)\}, t \in[0, T]$, is stable with stability constants $M \geq 1$ and $\omega=0$ (cf. 
Lemma 2), and the mapping $[0, T] \ni t \rightarrow A(t) x, x \in D(A)$, is continuously differentiable, then the family $\left\{\mathcal{A}_{n}(t)\right\}, t \in[0, T], n \in \mathbb{N}$, is uniformly stable, i.e., the following inequalities hold:

$$
\left\|\left(\lambda-\mathcal{A}_{n}\left(t_{k}\right)\right)^{-1}\left(\lambda-\mathcal{A}_{n}\left(t_{k-1}\right)\right)^{-1} \ldots\left(\lambda-\mathcal{A}_{n}\left(t_{1}\right)\right)^{-1}\right\| \leq \widetilde{M} \lambda^{-k}
$$

for $\lambda>0$, and

$$
\left\|\left(\lambda-\mathcal{A}_{n}\left(t_{1}\right)\right)^{-1}\left(\lambda-\mathcal{A}_{n}\left(t_{2}\right)\right)^{-1} \ldots\left(\lambda-\mathcal{A}_{n}\left(t_{k}\right)\right)^{-1}\right\| \leq \widetilde{M}(-\lambda)^{-k}
$$

for $\lambda<0$, for any finite sequence $0 \leq t_{1} \leq t_{2} \leq \ldots \leq t_{k} \leq T, k, n \in \mathbb{N}$, where $\widetilde{M} \geq 1$ is a constant independent of $n$.

Proof. The proof of this theorem is an analogue of that of Lemma 2.5 in [6] as well as Theorem 2.4 in [8, p. 133]. If $\lambda$ belongs to the resolvent set of the operator $\mathcal{A}_{n}(t)$, then it is easily seen that

$$
\left(\lambda-\mathcal{A}_{n}(t)\right)^{-1}=\left[\begin{array}{cc}
B_{n}^{-1}(t) & 0 \\
0 & I
\end{array}\right]\left(\lambda-\mathcal{B}_{n}(t)\right)^{-1}\left[\begin{array}{cc}
B_{n}(t) & 0 \\
0 & I
\end{array}\right]
$$

for $t \in[0, T], n \in \mathbb{N}$. By (70), for $\lambda>0$ we obtain

$$
\prod_{j=1}^{k}\left(\lambda-\mathcal{A}_{n}\left(t_{j}\right)\right)^{-1}=\prod_{j=1}^{k}\left[\begin{array}{cc}
B_{n}^{-1}\left(t_{j}\right) & 0 \\
0 & I
\end{array}\right]\left(\lambda-\mathcal{B}_{n}\left(t_{j}\right)\right)^{-1}\left[\begin{array}{cc}
B_{n}\left(t_{j}\right) & 0 \\
0 & I
\end{array}\right] .
$$

Letting, for $j=2, \ldots, k$,

$$
P_{n, j}:=\left(\left[\begin{array}{cc}
B_{n}\left(t_{j}\right) & 0 \\
0 & I
\end{array}\right]-\left[\begin{array}{cc}
B_{n}\left(t_{j-1}\right) & 0 \\
0 & I
\end{array}\right]\right)\left[\begin{array}{cc}
B_{n}^{-1}\left(t_{j-1}\right) & 0 \\
0 & I
\end{array}\right],
$$

we get

where

$$
\left[\begin{array}{cc}
B_{n}\left(t_{j}\right) & 0 \\
0 & I
\end{array}\right]\left[\begin{array}{cc}
B_{n}^{-1}\left(t_{j-1}\right) & 0 \\
0 & I
\end{array}\right]=\widetilde{I}+P_{n, j}
$$

is the unit matrix in $X \times X$.

$$
\widetilde{I}:=\left[\begin{array}{ll}
I & 0 \\
0 & I
\end{array}\right]
$$

From this we get

$$
\begin{gathered}
\prod_{j=1}^{k}\left(\lambda-\mathcal{A}_{n}\left(t_{j}\right)\right)^{-1}=\left[\begin{array}{cc}
B_{n}^{-1}\left(t_{k}\right) & 0 \\
0 & I
\end{array}\right]\left\{\left(\lambda-\mathcal{B}_{n}\left(t_{k}\right)\right)^{-1}\left(\widetilde{I}+P_{n, k}\right) \ldots\right. \\
\left.\ldots\left(\lambda-\mathcal{B}_{n}\left(t_{2}\right)\right)^{-1}\left(\widetilde{I}+P_{n, 2}\right)\left(\lambda-\mathcal{B}_{n}\left(t_{1}\right)\right)^{-1}\right\}\left[\begin{array}{cc}
B_{n}\left(t_{1}\right) & 0 \\
0 & I
\end{array}\right] .
\end{gathered}
$$

Using the uniform stability of the family $\left\{\mathcal{B}_{n}(t)\right\}$ (cf. Lemma 8), the norm of the expression in curly brackets in formula (77) may be estimated by

$$
2 M \lambda^{-k} \prod_{j=2}^{k}\left(1+2 M\left\|P_{n, j}\right\|\right) \text {. }
$$


Let us remark that

$$
\left\|P_{n, j}\right\| \leq\left\|\left[\begin{array}{cc}
B_{n}\left(t_{j}\right) & 0 \\
0 & I
\end{array}\right]-\left[\begin{array}{cc}
B_{n}\left(t_{j-1}\right) & 0 \\
0 & I
\end{array}\right]\right\|\left\|\left[\begin{array}{cc}
B_{n}^{-1}\left(t_{j-1}\right) & 0 \\
0 & I
\end{array}\right]\right\| .
$$

Now we estimate the norms of the matrices in (79). We have

$$
\begin{aligned}
\left\|\left(\left[\begin{array}{cc}
B_{n}\left(t_{j}\right) & 0 \\
0 & I
\end{array}\right]-\left[\begin{array}{cc}
B_{n}\left(t_{j-1}\right) & 0 \\
0 & I
\end{array}\right]\right)\left[\begin{array}{l}
y \\
x
\end{array}\right]\right\| \\
\quad=\left\|\left[B_{n}\left(t_{j}\right)-B_{n}\left(t_{j-1}\right)\right] y\right\| \\
\leq\left\|\left[B_{n}\left(t_{j}\right)-B_{n}\left(t_{j-1}\right)\right] B^{-1}(0)\right\| \cdot\|B(0) y\| \\
\leq \sup _{0 \leq t \leq T}\left[\left\|B_{n}^{\prime}(t) B^{-1}(0)\right\|\right]\left(t_{j}-t_{j-1}\right)\|y\|_{Y} .
\end{aligned}
$$

By Lemma 5, from the above it follows that

$$
\left\|\left[\begin{array}{cc}
B_{n}\left(t_{j}\right) & 0 \\
0 & I
\end{array}\right]-\left[\begin{array}{cc}
B_{n}\left(t_{j-1}\right) & 0 \\
0 & I
\end{array}\right]\right\| \leq L\left(t_{j}-t_{j-1}\right)
$$

where $L:=\sup \left\{\left\|B_{n}^{\prime}(t) B^{-1}(0)\right\|: t \in[0, T], n \in \mathbb{N}\right\}$ is a constant independent of $t \in[0, T]$ and of $n \in \mathbb{N}$ (cf. (56)).

Since the second factor on the right-hand side of (79) is, by virtue of (64), estimated by $\gamma$, which does not depend on $n$ either, we get

$$
\left\|P_{n, j}\right\| \leq K\left(t_{j}-t_{j-1}\right), \quad j=2, \ldots, k,
$$

where $K=L \gamma$.

Using the estimates (64), (65), (80) and the elementary inequality $1+x \leq$ $e^{x}$ for $x \geq 0$, from (77) we obtain

$$
\begin{aligned}
\left\|\prod_{j=1}^{k}\left(\lambda-\mathcal{A}_{n}\left(t_{j}\right)\right)^{-1}\right\| & \leq 2 M b \gamma|\lambda|^{-k} \prod_{j=2}^{k} \exp \left(2 M K\left(t_{j}-t_{j-1}\right)\right) \\
& \leq 2 M b \gamma \exp (2 M K T)|\lambda|^{-k} .
\end{aligned}
$$

Therefore

$$
\left\|\prod_{j=1}^{k}\left(\lambda-\mathcal{A}_{n}\left(t_{j}\right)\right)^{-1}\right\| \leq \widetilde{M}|\lambda|^{-k},
$$

where $\widetilde{M}=2 M b \gamma \exp (2 M K T)$ is a constant independent of $n \in \mathbb{N}$.

Analogously we can prove the estimate (74).

Because the stability constants $\widetilde{M}$ and $\omega=0$ do not depend on $n \in \mathbb{N}$, this implies that the sequence $\left\{\mathcal{A}_{n}(t)\right\}$ is uniformly stable (see [1]). Moreover, each operator $\mathcal{A}_{n}(t)$ for fixed $t \in[0, T]$ and $n \in \mathbb{N}$ is the infinitesimal generator of a strongly continuous group.

Now we can prove the following important theorem. 
TheOREM 4. Let assumptions $\left(\mathrm{Z}_{1}\right)-\left(\mathrm{Z}_{4}\right)$ hold. If $\{A(t)\}, t \in[0, T]$, is a stable family with stability constants $M \geq 1$ and $\omega=0$, and the mapping $[0, T] \ni t \rightarrow A(t) x, x \in D(A)$, is continuously differentiable, then the conditions of Theorem 1 are satisfied for the operator $\mathcal{A}(t)$ given by (60) and the operators $\mathcal{A}_{n}(t)$ constructed according to formula (61).

P r o of. The stability of $\{A(t)\}$ yields the estimate (23), and so, by Theorem 2 , we get assumptions $1^{\circ}$ and $2^{\circ}$ of Theorem 1 . Let $\mathcal{V}_{n}(t, s)$ denote the evolution operator (fundamental solution) corresponding to the operator $\mathcal{A}_{n}(t)$. From Lemmas 2 and 8 and Theorem 3 it follows that the approximating sequence $\left\{\mathcal{A}_{n}(t)\right\}$ is uniformly stable with stability constants $\widetilde{M}$ and $\omega=0$ (see (81)). By Theorem 1 of [1] we obtain

$$
\left\|\mathcal{V}_{n}(t, s)\right\| \leq \widetilde{M} \quad \text { for } t, s \in[0, T], n \in \mathbb{N} .
$$

Basing on the estimate (82), analogously to the proof of Theorem 3.11 in [7, p. 208] we can prove that $\left\{\mathcal{V}_{n}(t, s)\right\}$ is strongly and uniformly convergent to $\mathcal{V}(t, s)$ in $K_{T}=[0, T] \times[0, T]$. We omit the details.

From this we have, as a consequence of Theorem 1, the following corollary.

COROLlaRY 2. Under the assumptions of Theorem 4 the Cauchy problem for the equation

$$
\frac{d w}{d t}=\mathcal{A}(t) w, \quad t \in[0, T],
$$

is uniformly correct and $\mathcal{V}(t, s)$ is the evolution operator corresponding to it.

By definition, $\mathcal{A}(t)$ for each $t \in[0, T]$ is a linear operator acting from $Y \times X$ into itself, where $Y$ is the space $D(B)$ with graph norm (see (59)). Therefore, the evolution operator $\mathcal{V}(t, s)$ may be written as

$$
\mathcal{V}(t, s)=\left[\begin{array}{ll}
C(t, s) & S(t, s) \\
b(t, s) & a(t, s)
\end{array}\right]
$$

Let us define (cf. [10] and [6]) the operator

$$
S(t, s) x:=\Pi_{1} \mathcal{V}(t, s)\left[\begin{array}{l}
0 \\
x
\end{array}\right] \quad \text { for every } x \in X,
$$

where $\Pi_{1}\left[\begin{array}{l}y \\ x\end{array}\right]:=y$ for $y \in Y, x \in X$. Using Definition 3 and formula (85) one can prove that $S(t, s)$ is the evolution operator (fundamental solution) for equation (57). For details see [6].

Summing up, we can formulate the main result of this paper.

THEOREM 5. If the assumptions of Theorem 4 are satisfied, then the Cauchy problem for equation (57) is uniformly correct and the $S(t, s)$ defined by formula (85) is the evolution operator corresponding to it. 
If the fundamental solution $S(t, s)$ for $(57)$ is known and if the function $f:[0, T] \rightarrow X$ satisfies certain conditions, and if $u_{0}, u_{1} \in D(A)$, then the problem (7) has the unique solution $u$ given by

$$
u(t)=-\left.\frac{\partial}{\partial s} S(t, s)\right|_{s=0} u_{0}+S(t, 0) u_{1}+\int_{0}^{t} S(t, s) f(s) d s
$$

(see for example [6] and [11]).

\section{References}

[1] J. Bochenek and T. Winiarska, Evolution equations with parameter in the hyperbolic case, Ann. Polon. Math. 64 (1996), 47-60.

[2] H. O. Fattorini, Ordinary differential equations in linear topological spaces, I, J. Differential Equations 5 (1968), 72-105.

[3] -, Ordinary differential equations in linear topological spaces, II, ibid. 6 (1969), $50-70$.

[4] - Second Order Linear Differential Equations in Banach Spaces, North-Holland, New York, 1985.

[5] T. Kato, Perturbation Theory for Linear Operators, Grundlehren Math. Wiss. 132, Springer, New York, 1980.

[6] M. Kozak, A fundamental solution of a second-order differential equation in a Banach space, Univ. Iagel. Acta Math. 32 (1995), 275-289.

[7] S. Krein, Linear Differential Equations in Banach Space, Amer. Math. Soc., 1972.

[8] A. Pazy, Semigroups of Linear Operators and Applications to Partial Differential Equations, Appl. Math. Sci. 44, Springer, 1983.

[9] H. Tanabe, Equations of Evolution, Pitman, London, 1979.

[10] C. C. Travis and G. F. Webb, Cosine families and abstract nonlinear second order differential equations, Acta Math. Acad. Sci. Hungar. 32 (1978), 75-96.

[11] T. Winiarska, Evolution equations of second order with operator depending on $t$, in: Selected Problems of Mathematics, Cracow University of Technology, Anniversary issue, 1995, 299-311.

[12] K. Yosida, Functional Analysis, Springer, New York, 1980.

Institute of Mathematics

Technical University of Kraków

Warszawska 24

31-155 Kraków, Poland

E-mail: u-2@institute.pk.edu.pl 